\title{
Example telescope simulations with the AstroX telescope toolbox for McXtrace
}

Knudsen, Erik B.; Svendsen, Sara; Henriksen, Peter L.; Jegers, Arne S.; Willendrup, Peter K.; Ferreira, Desiree D. M.

Published in:

Space Telescopes and Instrumentation 2020: Ultraviolet to Gamma Ray

Link to article, DOI:

$10.1117 / 12.2561372$

Publication date:

2020

Document Version

Publisher's PDF, also known as Version of record

Link back to DTU Orbit

Citation (APA):

Knudsen, E. B., Svendsen, S., Henriksen, P. L., Jegers, A. S., Willendrup, P. K., \& Ferreira, D. D. M. (2020). Example telescope simulations with the AstroX telescope toolbox for McXtrace. In J-W. A. den Herder, S.

Nikzad, \& K. Nakazawa (Eds.), Space Telescopes and Instrumentation 2020: Ultraviolet to Gamma Ray (Vol. 11444). [114443Q] SPIE - International Society for Optical Engineering. Proceedings of SPIE - The International Society for Optical Engineering https://doi.org/10.1117/12.2561372

\section{General rights}

Copyright and moral rights for the publications made accessible in the public portal are retained by the authors and/or other copyright owners and it is a condition of accessing publications that users recognise and abide by the legal requirements associated with these rights.

- Users may download and print one copy of any publication from the public portal for the purpose of private study or research.

- You may not further distribute the material or use it for any profit-making activity or commercial gain

- You may freely distribute the URL identifying the publication in the public portal 


\section{Example telescope simulations with the AstroX telescope toolbox for McXtrace}

Knudsen, Erik, Svendsen, Sara, Henriksen, Peter, Jegers, Arne, Willendrup, Peter, et al.

Erik B. Knudsen, Sara Svendsen, Peter L. Henriksen, Arne S. Jegers, Peter K. Willendrup, Desirée D. M. Ferreira, "Example telescope simulations with the AstroX telescope toolbox for McXtrace," Proc. SPIE 11444, Space Telescopes and Instrumentation 2020: Ultraviolet to Gamma Ray, 114443Q (13 December 2020); doi: 10.1117/12.2561372

SPIE. Event: SPIE Astronomical Telescopes + Instrumentation, 2020, Online Only 


\title{
Example telescope simulations with the AstroX telescope toolbox for McXtrace.
}

\author{
Erik B. Knudsen ${ }^{a}$, Sara Svendsen ${ }^{b}$, Peter Lindquist Henriksen ${ }^{b}$, Arne S. Jegers ${ }^{b}$, Peter K. \\ Willendrup $^{a}$, Desiree D. M. Ferreira ${ }^{b}$ \\ $a$ DTU Fysik, Technical University of Denmark, Fysikvej, bygning 311, 2800, Kgs. Lyngby, Denmark \\ $b$ DTU Space, Technical University of Denmark, Elektrovej, bygning 327, 2800, Kgs. Lyngby, Denmark
}

\begin{abstract}
We present a number of example studies of telescope optics using the latest version of the AstroX add on toolbox for McXtrace. Among which are first, a benchmark study of effective area and vignetting for the Chandra X-ray Observatory which we find to match well with literature. Second, a convenient way of building a telescope model (in this case NuSTAR) with many similar optical elements scripted using python module. This lends itself well to be included in online notebooks and/or for teaching. Third, we show a new AstroX module for lobster eye optics, and fourth, a study of the proposed solar axion telescope BabyIAXO.
\end{abstract}

Keywords: X-ray, telescope, simulation, optics, ray-tracing

\section{INTRODUCTION}

In a Wolter telescope optic ${ }^{1}$ the basic geometry is a curved mirror (fig. 1). In the transversal plane, perpendicular to the optical axis, the mirrors are curved along a simple cylinder, whereas along the optical axis the mirrors form (in a true Wolter I geometry) pairs with first parabolic and hyperbolic curvatures. There are many variations on this scheme, in which the parabolic and/or hyperbolic curvature is replaced by a similar curvature, ${ }^{2-4}$ e.g. to approximate the curvature with something more production friendly, such as the conical approximation, or to target some different optical characteristic. To exemplify: the true Wolter I geometry is optimal for onaxis focusing capabilities, whereas a Wolter-Schwarzschild-geometry improves the off-axis characteristics at the expense of on-axis performance.

The small incidence angle of telescope mirrors that is necessary in the X-ray regime has some important implications; The area-coverage of a single mirror pair is very small. Further, the mirror becomes very sensitive to deviations from the ideal shape or figure. To address the former, X-ray telescopes optics are in general assemblies of several mirrors. For instance, in a Wolter optic mirror come in pairs in a shell structure (fig. 1). This obviously requires a support structure holding the mirrors in place, which in turn implies that some fraction of the ideal area of coverage becomes obscured. The second issue has many facets to it, depending on the nature of the deviations from the ideal profile. For instance (from a geometrical optics point of view), high spatial frequency, $\lambda_{e, m} \ll \lambda_{\mathrm{x}-\mathrm{ray}}$, imperfections (roughness) tend to lower the overall reflectivity of the surface, whereas low frequency components (figure and slope errors) alter the surface normal through which optical rays should be reflected. Intermediary frequencies $\left(\lambda_{e, m} \approx \lambda_{\mathrm{x} \text {-ray }}\right)$ may introduce interference effects to the reflection pattern. In addition, in a realized telescope, shells may be displaced and/or misoriented. All of these effect illustrate the need for optimization and robustness studies in the design process, and hence a need for practical simulation tools to perform such studies.

AstroX is a tool for this task, which is also general such that it may be reused for many telescopes and missions. AstroX is an extension to the McXtrace ${ }^{5}$ software package - an X-ray tracing framework designed to perform optimization simulations for terrestrial synchrotron and/or XFEL beamlines. As is AstroX, McXtrace itself is an open source project which is general, modular, and extensible. Hence, it lends itself well to the task.

We will in this paper focus on some of the new developments in AstroX/McXtrace, including a lobster eye optics model, a model for including a grating in a telescope as well as a couple of example of AstroX/McXtrace usage: A study of a proposed (but not yet final) design of the BabyIAXO ${ }^{6}$ mission, as well as studies of the long standing NuSTAR ${ }^{7}$ and Chandra $^{8}$ missions for bench-marking, illustrative purposes.

Space Telescopes and Instrumentation 2020: Ultraviolet to Gamma Ray, edited by Jan-Willem A. den Herder Shouleh Nikzad, Kazuhiro Nakazawa, Proc. of SPIE Vol. 11444, 114443Q · C 2020 SPIE CCC code: $0277-786 \mathrm{X} / 20 / \$ 21 \cdot$ doi: $10.1117 / 12.2561372$

Proc. of SPIE Vol. 11444 114443Q-1 


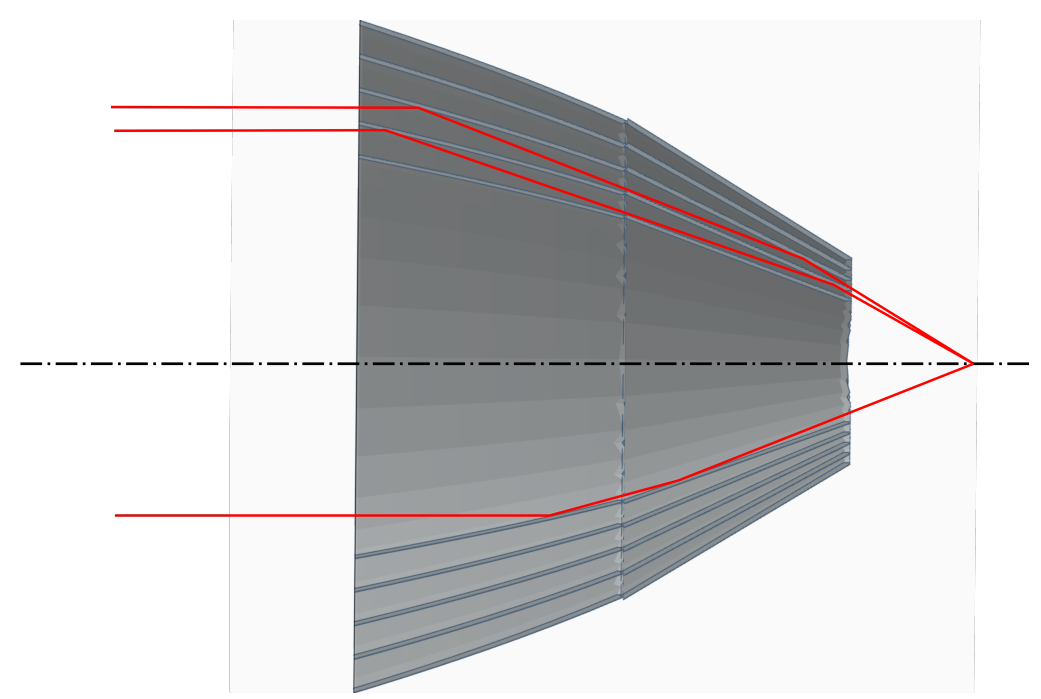

Figure 1. Sketch of a Wolter I optic. Concentric shells reflect rays in two confocal mirrors, parabolic and hyperbolic respectively.

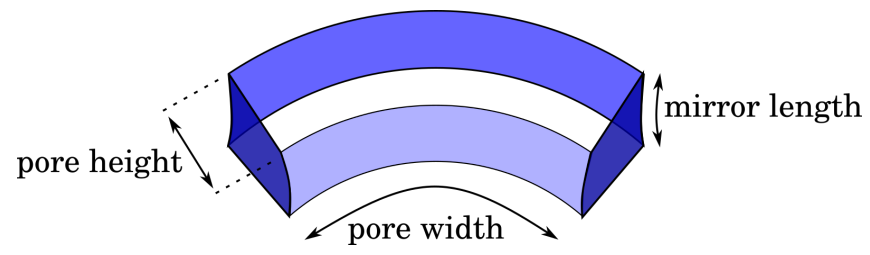

Figure 2. Sketch of a pore through which photons are "guided". The Pore is viewed from upstream as seen from the source. For clarity of display the mirror length is short and the height is larger than would be likely in a real case. Since the downstream transversal curvature radius is smaller than upstream the side walls are not straight lines, if the pore width is constant.

\section{METHOD}

\subsection{The pore model and algorithm}

One of the most basic optical building blocks of the AstroX system is the "Pore". It constitutes a channel, walled on four sides (See fig. 2). The outer and inner (wrt. the optical axis) walls are curved along their lengths to mimic a Wolter mirror, whereas the side walls run along the length of the channel. No restriction is imposed on the width of a pore (apart from the trivial: $w<2 \pi r$ ). A single mirror may therefore be modelled by removing the side walls. Along the same lines the inner surface of the pore may be shifted inwards to the optical axis to make the pore behave as a single, free-floating, mirror. In theory, an entire, large-scale, SPO-based optic like the one planned for the ATHENA mission, ${ }^{9,10}$ could be built from pores. This would ensure complete generality and flexibility in exploring misalignment/misorientation effects as well as the option to construct completely heterogeneous optical assemblies. This is however impractical for larger scale optics as it involves many thousands of pores. To some extent this may be counteracted by scripting ${ }^{11}$ as McXtrace and hence AstroX relies on a structured text-file for its optics description, which can be written by external means. A harder memory limit will be reached when the c-compiler back-end cannot handle the sheer volume of objects. We note that this problem will be alleviated in the upcoming generation of McXtrace code generator ${ }^{12}$ with which AstroX-users also gain the ability to run simulations on GPUs, where large scale speed-ups may be found for long simulation runs.

By generalization of the pore module we may arrive at other Wolter-building blocks: The shell, ring, and Mirror Module (MM) constructs. A Shell is a circumference-wide mirror with no walls, but which has an inner wall. A stack of identical shells constitutes a ring, and similarly a radial and azimuthal stack of identical pores is a mirror module. The notion of a mirror module has been borrowed from the ATHENA-project. ${ }^{13}$ 


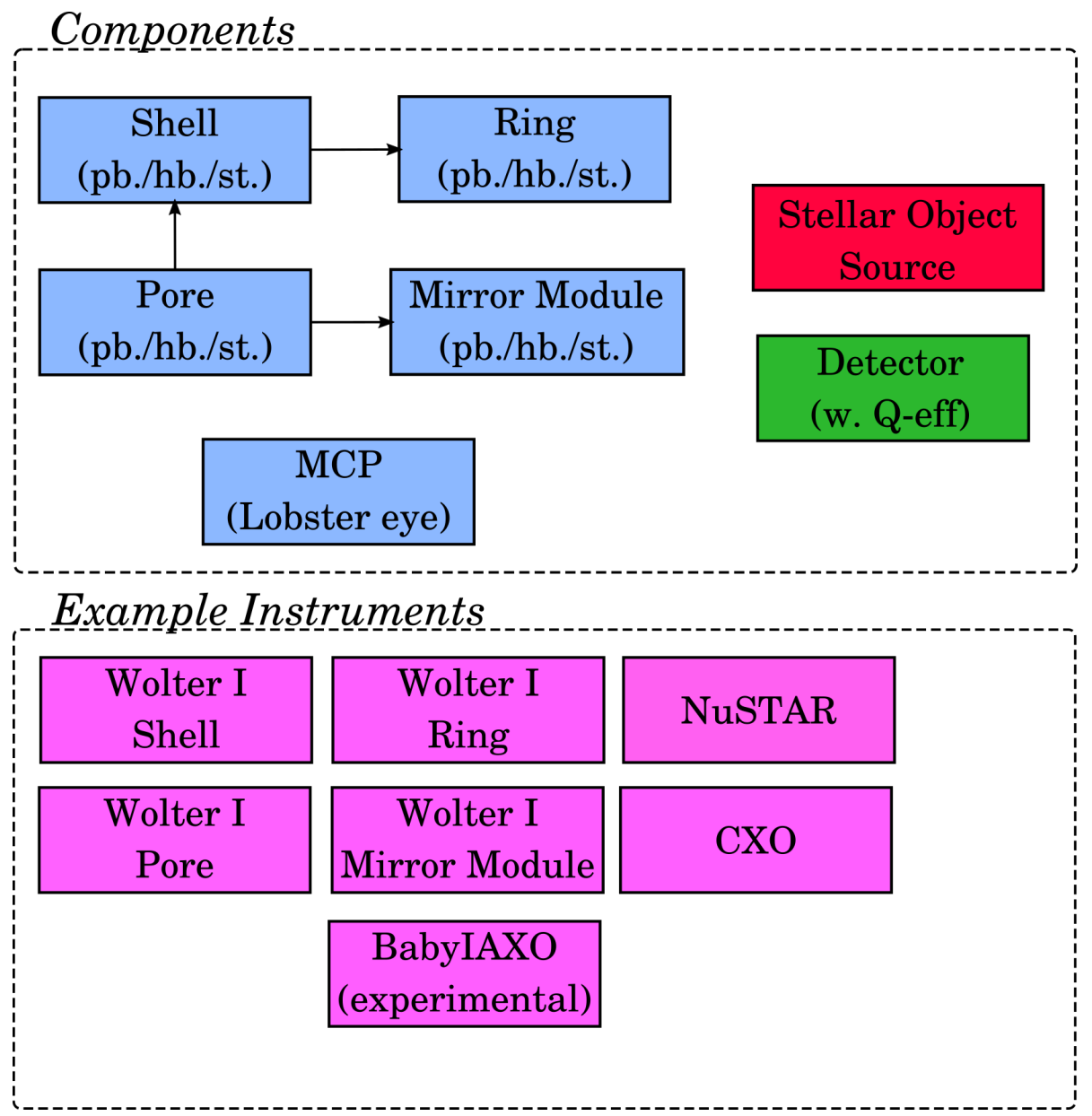

Figure 3. Contents of the AstroX/McXtrace library. Optical devices in the broad sense are known as "Components" whereas an arrangements of components constitute "Instruments". Note that, in addition, all component within McXtrace are also automatically available to AstroX-users.

\subsection{Specializations of the pore model, and their characteristics}

Having established the most basic building block of a Wolter-style optic, we now focus on some practical extensions thereof, namely the Shell, Ring, and Mirror Module. The Shell is simply a full circle pore with no side walls, the ring is a radial set of mirror plates of same length, and the mirror module is a grid of pores of equal size extending in both the radial and azimuthal direction of the Wolter optics. The latter is naturally the main building block of the ATHENA-telescope. The current state of the AstroX/McXtrace library is reflected in fig. 3. Here we find the Wolter optics constituents mentioned above. Furthermore there are examples of use cases, among them those used to generate the results reported below (section 3).

\subsection{Lobster eye optic}

We now focus our attention on a different type of telescope, the lobster eye based kind. ${ }^{14,15}$ A lobster eye, consists of a set of elongated channel set on a spherical surface. This creates a common focal point for all channels at a distance of half the radius of the spherical surface (fig. 4). From a ray tracing algorithm point of view, this is very similar to a an SPO-based optic, in the sense that optical rays travel through a channel with four walls which may be coated with a reflecting surface. The geometry and assembly of the channels being the differences. 


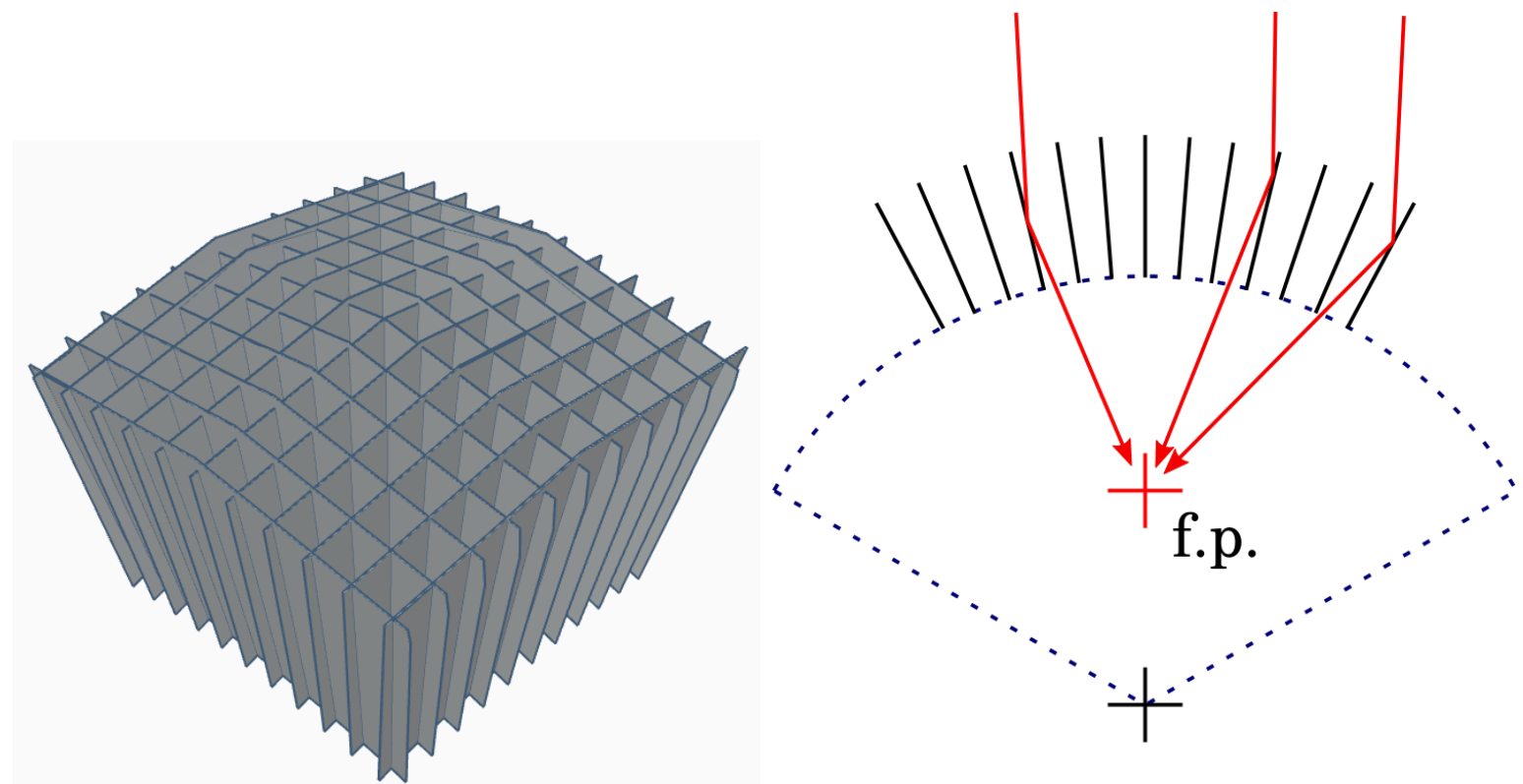

Figure 4. Schematics of a lobster eye optic. Left: a 3D rendering of a lobster eye. Right: 1D-cutout of the optic. N.b. while only a single reflection is indicated in the drawing - for $2 \mathrm{D}$-focusing two are required.

\subsection{Extracting optical characteristics}

Some of the first parameters to be requested from a model of a telescope optics are Effective Area $\left(A_{\text {eff }}\right)$ and Vignetting function. It is therefore of interest to describe how these may be extracted from Monte Carlo ray tracing studies.

\subsubsection{Effective Area}

Extracting the effective area from a telescope optic can be done in several ways There are at least two ways of computing the effective are of a telescope using McXtrace. In general the effective area is the collecting area of the telescope corrected downward by the reflectivities of the reflecting objects. For a system consisting of simulations of single objects (such as SPO mirror modules or single shells) it is possible to compute the effective area as:

$$
A_{e f f}=\sum_{i}^{N} A_{i}\left\langle R_{p a} R_{h y}\right\rangle
$$

where $\left\langle R_{p a} R_{h y}\right\rangle$ is the weighted mean of parabolic- and hyperbolic reflectivities multiplied, encountered by the rays, and $A_{i}$ is the collecting area of the $i$ :th optical object. If the collecting area of all objects are known, we may use two instances of the general purpose component Monitor_nD from McXtrace to directly measure the mean of the encountered reflectivities.

The collecting area can sometimes be non-trivial (or tedious) to calculate. In such a case an alternative procedure can be used: Assuming that the telescope is fully illuminated by a wavefront. For practical calculations we have to limit ourselves to emitting photons into an area $\mathbf{B}$ which fully covers the collecting area A. Place an ideal monitor before and after the optic. If the optic transports all collected photons to the monitor behind, then we have:

$$
\frac{\mathbf{A}}{\mathbf{B}}=\frac{I_{A}}{I_{B}} \Rightarrow \mathbf{A}=\mathbf{B} \frac{I_{A}}{I_{B}}
$$

where $I_{B}$ and $I_{A}$ denote the recorded intensities by the "before" and "after" monitors. Let $I_{A^{*}}$ be the intensity recorded in a monitor after the optic, including reflectivity effects, side-wall losses if relevant etc. The reflectivity effects can be found as:

$$
\left\langle R_{p} a R_{h} y\right\rangle=\frac{I_{A^{*}}}{I_{A}}
$$


Combining the effective area for a single optical object (analogous to before) with eqs. (2) and (3) we get:

$$
A_{e f f}=\mathbf{A}\left\langle R_{p} a R_{h} y\right\rangle=\mathbf{A} \frac{I_{A^{*}}}{I_{A}}=\mathbf{B} \frac{I_{A}}{I_{B}} \frac{I_{A^{*}}}{I_{A}}=\mathbf{B} \frac{I_{A^{*}}}{I_{B}}
$$

By summing over all optical objects we may get the full effective area for the telescope.

$A_{\text {eff }}$ is generally a function of energy. In the first case, we may measure the encountered mean square reflectivity $\left\langle R_{p a} R_{h y}\right\rangle$ as a function of energy and weight by the collecting area in a simple post-processing step. In the second case the effective area may be directly extracted; as long as we catch all the rays in our integrating monitors, i.e. they are large enough, we can directly measure $I_{B}(E)$ and $I_{A^{*}}(E)$ energy resolved. In McXtrace terms: two instances of Emonitor. B is a known, and often constant, input parameter to the source model. Thus, the energy resolved effective area can quite easily be computed. As eq. (4) does not explicitly include the energy dependence we may extend the procedure to other independent variables, such as incoming divergence. In AstroX/McXtrace we can do this by temporarily storing the incoming divergence for each ray only to measure the transmitted ray as a function of stored divergence after the ray has passed through the telescope optic. Just as for the effective area, we thus obtain a direct handle on the vignetting function.

\section{RESULTS}

\subsection{NuSTAR-like telescope}

As a first example we have built a model of the existing telescope NuSTAR. ${ }^{16,17}$ For sure, more accurate models exists $^{18-20}$ but as a first step illustrating the possibilities of AstroX it serves well. The model we have adopted here simply regards the telescope as a set of shell mirrors with a set of obscuring spokes in front and behind the optic. The optic is a conical approximation of a Wolter optic with $\mathrm{Pt} / \mathrm{C}$ and $\mathrm{W} / \mathrm{Si}$ coatings on the inner and outer array structures respectively. The coatings themselves have been optimized for each plate (or rather in groups of plates), taking the radial coordinate and associated grazing angle into account. ${ }^{21}$ Defining the complete telescope model with all its 133 layers by hand, is a tedious and error prone procedure. An easy way of sidestepping this issue is by the (as yet experimental) python scripting layer: McXtrace_script developed as part of the VINYL-project, ${ }^{11}$ where the entire NuSTAR optic may be defined in $\approx 70$ lines of code (see listing 1 ). Furthermore this way of running simulations lends itself very well to the use of Jupyter notebooks for on-line training. ${ }^{22}$

Listing 1. Snippet of source code for generating a simulation with $2 \times 133$ mirror shells mimicking NuSTAR. The code shown is the main loop that generates the mirror that form the bulk of the simulation code.

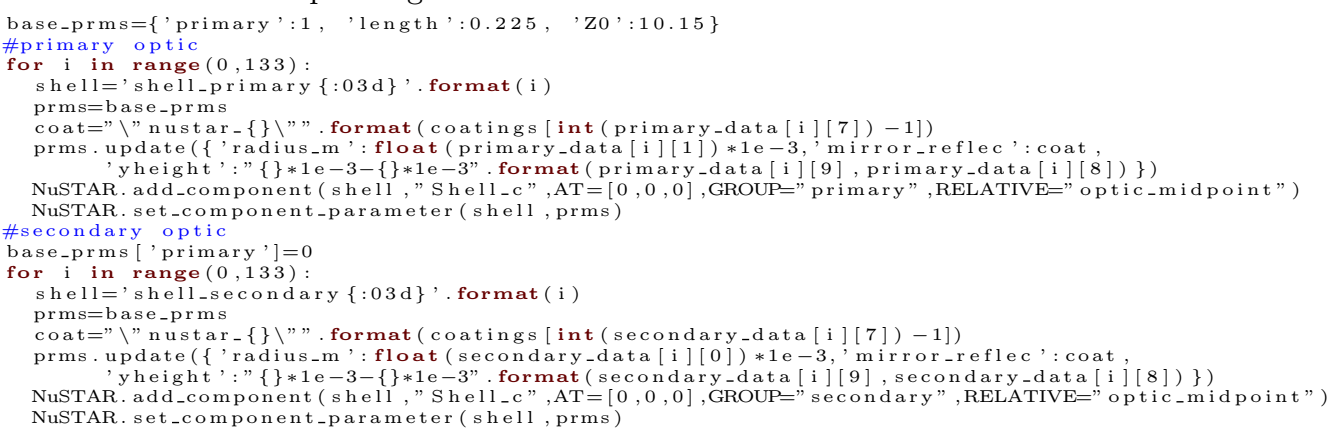

Figure 5 shows the transverse intensity distribution for the NuSTAR model immediately after the optic and at the focal plane. We find that the model behaves as expected given that no support spokes are included in this minimal model.

\subsection{Chandra X-ray telescope}

Our next example is a model of the Chandra X-ray Observatory (CXO). Arguably yet another ray tracing model of this telescope is superfluous but nevertheless serves as an excellent example of what is easily achievable with the AstroX/McXtrace toolbox, and also to serve as a benchmark. First of all, we use the procedure outlined 

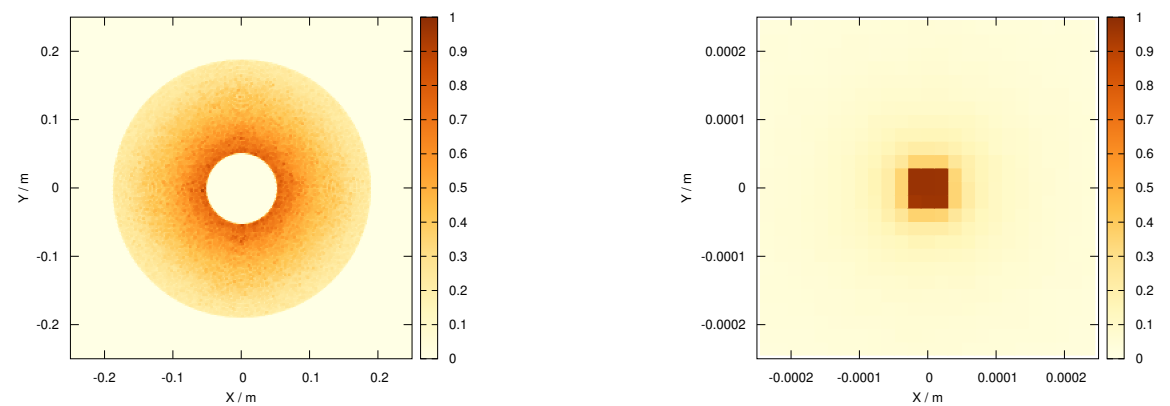

Figure 5. Transverse intensity distribution after the NuSTAR optics when illuminated by a uniformly distributed, perfectly collimated, source with $E \in[0.1,70] \mathrm{kev}$. Immediately after the optic (left) and at the focal plane (right).

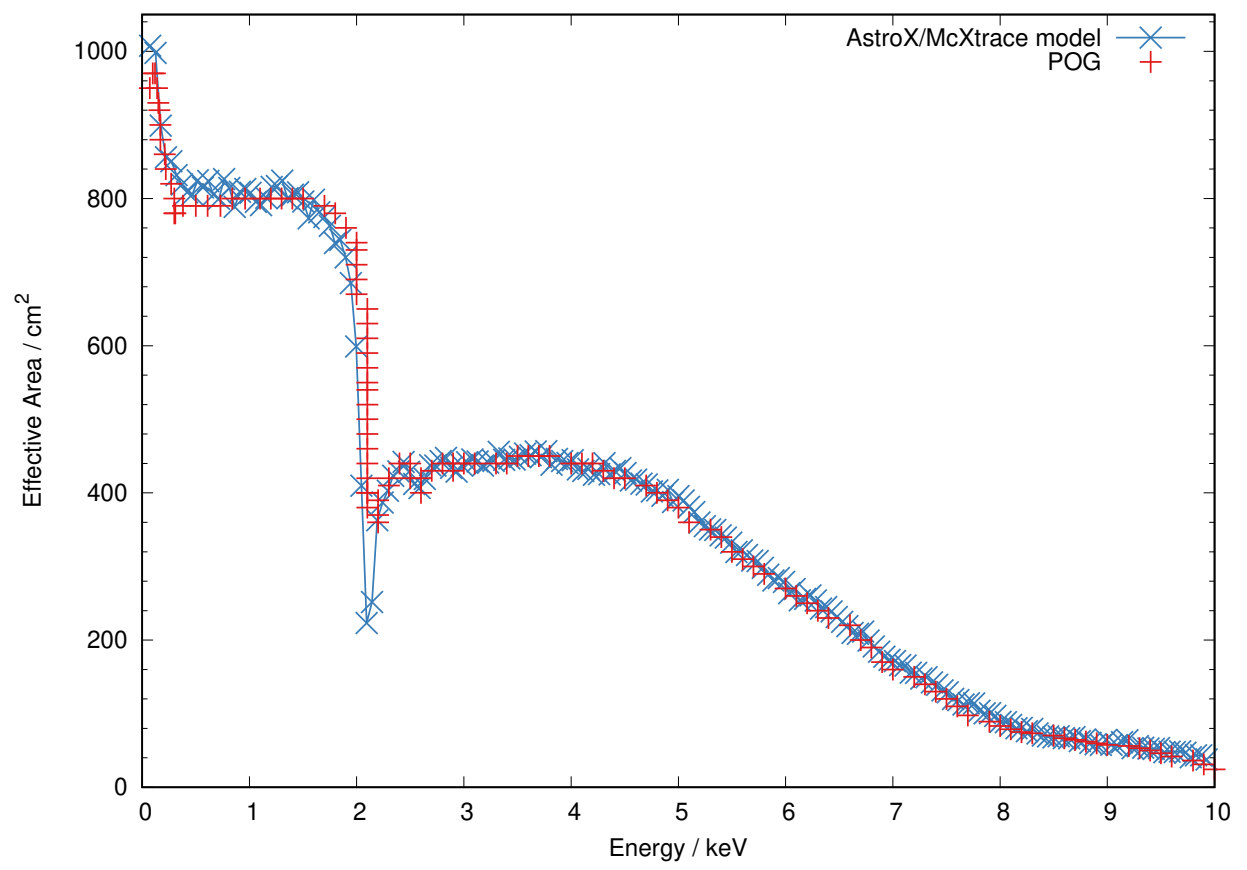

Figure 6. Effective area as a function of energy for an ideal model of the Chandra X-ray Observatory. Blue is the AstroX/McXtrace model, Red is the effective area extracted from the Proposers' Observatory Guide (POG). ${ }^{24}$

above to compute the effective area of the telescope for a range of energies. First we computed the coating reflectivity in the relevant range of energies and grazing angles using IMD. ${ }^{23}$ Second we follow the procedure outlined above to get at the effective area (see fig. 6.). We find the results to agree very well with those reported in the Chandra Proposers' Guide. ${ }^{24}$ In this idealised model there is no modelling of contamination, nor other surface imperfections that affect the effective area. Such effects can and should be included in the model, but is considered out of scope for this report.

As noted before (see section 2.4.1), if our monitors are instead set to resolve the computed intensity in incoming divergence, or off-axis angle, we may directly measure the vignetting function of the telescope, by expressing the effective area as function of incoming divergence. In AstroX/McXtrace terms this is done using the general purpose monitor: Monitor_nD, which may be used to measure intensity (or indeed any signal) as a function of anything. Figure 7 shows the effective area of the AstroX/McXtrace CXO as a function of off-axis entry angle for a range of energies. As before we find these results to match those reported in ref. 24 . 


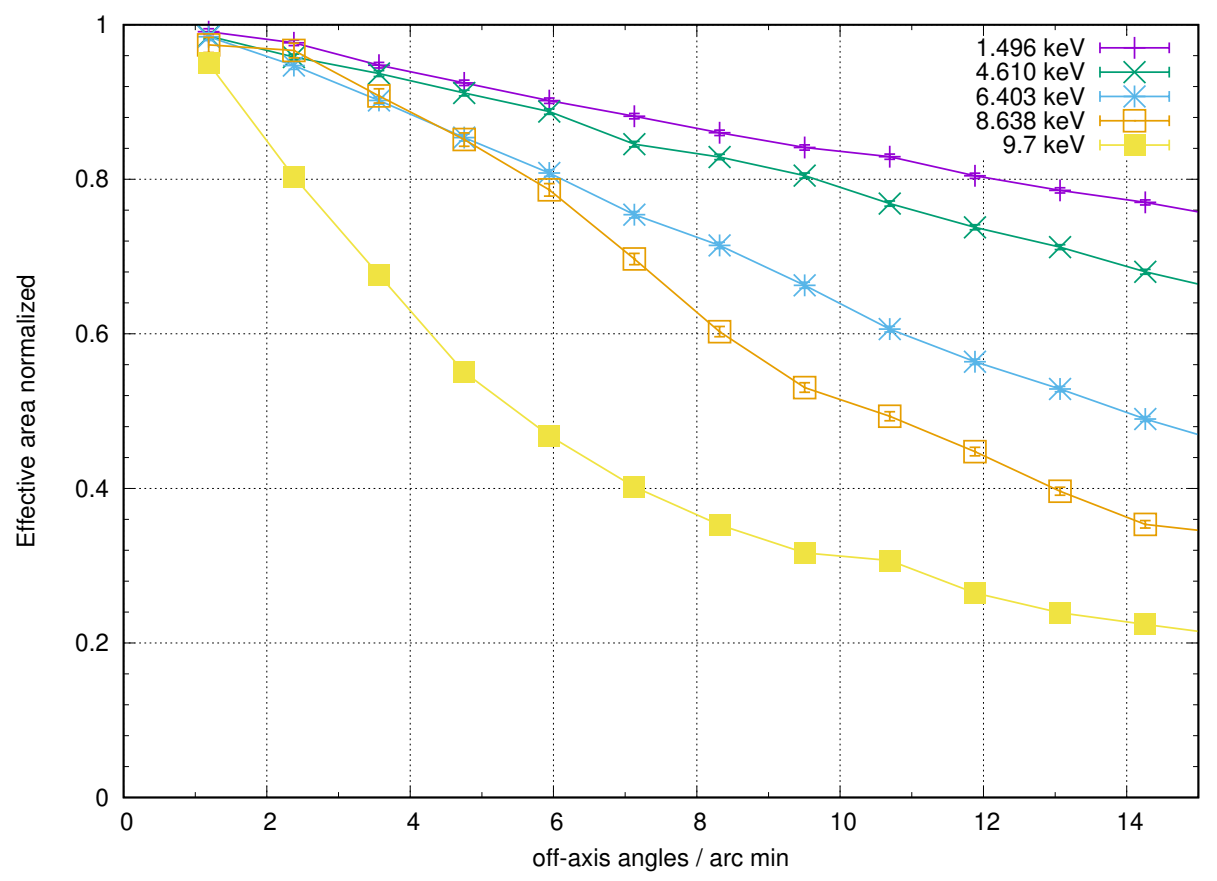

Figure 7. Effective area as a function of off-axis angle for a range of energies. The energies are chosen to correspond to figure 4.2 in ref. 24 .
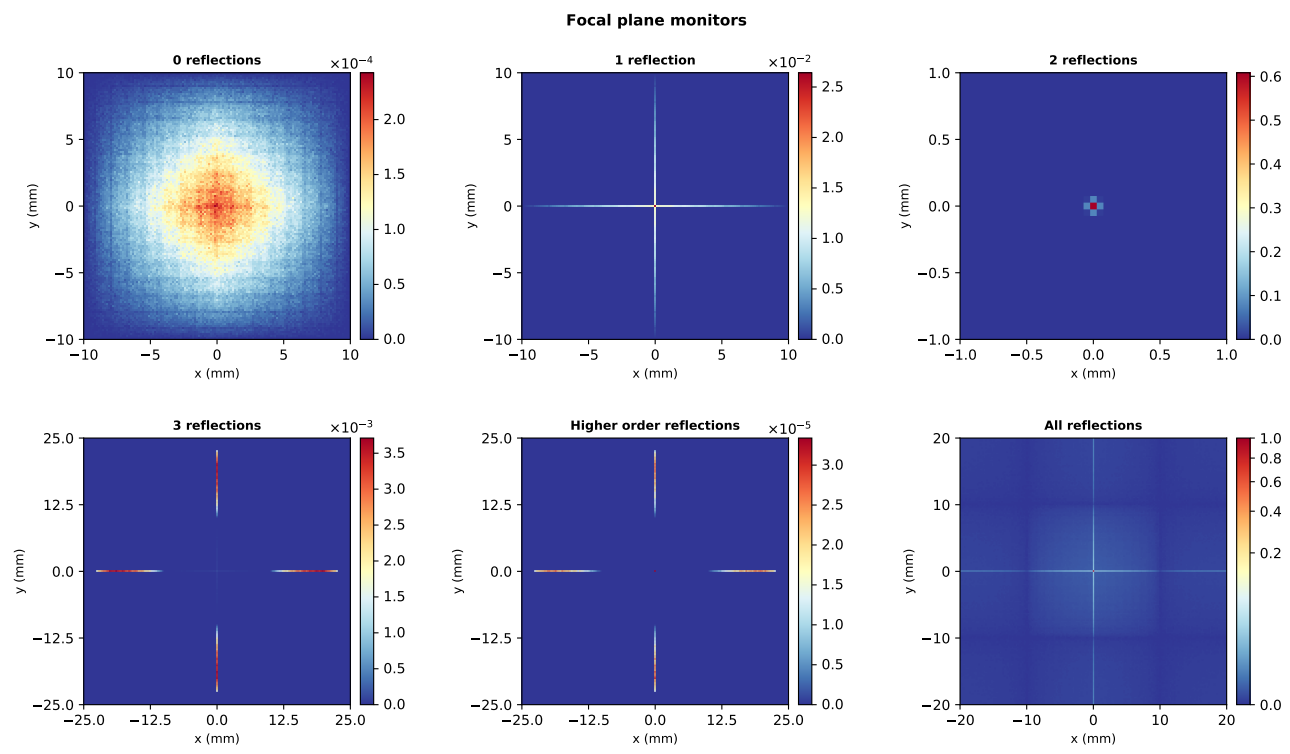

Figure 8. Intensity pattern recorded at the focal plane of a lobster-eye optic with radius, thickness etc. for varying numbers of reflections in the channels.

\subsection{Lobster-eye optic-based telescope}

AstroX/McXtrace allows to flag rays with the number of reflections that have occurred inside the channel. Doing so we may separate the focal plane response of the lobster eye optics into its constituent parts as is done in fig. 8. A single set of square shaped channels are illuminated on-axis with a uniform energy interval $E \in[0.3,6] \mathrm{keV}$. We divide the recorded intensity into reflection orders. As expected, only the 2nd order reflections contribute significantly to the central spot, whereas the line-shapes come from odd and higher order reflections. It is clear 


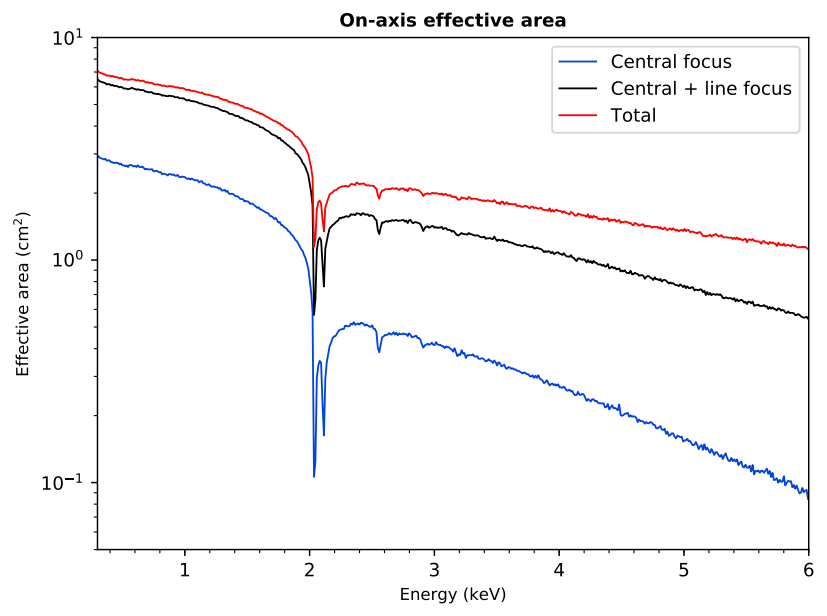

Figure 9. Effective area of a lobster eye optics as a function of energy.

that this should be so, from considering the square shape of the channels; 1st order can only be focused in one dimension, the one whose principal axis runs parallel to the plane of reflection. For orders $>2$ the radiation is overfocused. Furthermore, as the grazing angle increases with order, the associated reflectivity decreases. Thus, higher order reflection become less significant. This is clearly reflected in the effective area of the lobster eye as shown in fig. 9. Qualitatively we find these results in agreement with previously reported results for similar study. ${ }^{25}$

\subsection{BabyIAXO model}

As a last example we present yet another Wolter optics based telescope; a study of the proposed BabyIAXO telescope. Our model should be thought of a as work in progress, as the design of the telescope has not yet been finalized. ${ }^{6}$ In this case the optical setup consists partially of a true Wolter I (the outer part) and partially of a conical approximation to the Wolter I optic (the inner part). A brief test of the focusing properties (fig. 10) shows the optics behaves as expected. Here we have specifically included a set of support spokes that obscure part of the telescope optics. Although this study is not the finalized structure, it nonetheless shows a convenient way of to model the response of a plausible set of spokes. The radiation impinging on the optics has a divergence roughly matching that of the sun: $\approx 0.9 \mathrm{mrad}$, meaning the spoke structure is only visible just after the optics exit.

Unsurprisingly, at the upper end of the energy range only the inner modules contribute significantly to the recorded signal - a fact that is also clear from studying the effective area shown in in fig. 11. We may separate the signal coming from the two parts of the telescope: inner, conical approximation and outer, true Wolter type I, and compute the effective area for each of the two. Figure 11 shows just that, the effective area for the two optical parts separately, and together. We find the cut-off energy above which the outer telescope is largely inactive to be $\approx 8 \mathrm{keV}$.

\section{DISCUSSION}

The system of optical elements that makes up AstroX/McXtrace covers most styles of optics relevant for X-ray telescope optics, yet is not complete. To cover even more of existing and proposed instrumentation, more optical elements need to pulled in. One important aspect is the use of gratings to separate energy ranges. Here we may rely on models built for reflection and transmission gratings within the larger frame of McXtrace. In fact we speculate that a tighter integration or inclusion of AstroX is beneficial to both the telescope and terrestrial science communities. One quite obvious point is to build models of calibration and testing facilities such as PANTER. ${ }^{26}$ Such a model could enable design of experiments and facilities, along the lines of what is being done for beamlines. ${ }^{27,28}$ Many other software packages are available to users for telescope calculations, but this coupling is fairly unique. 

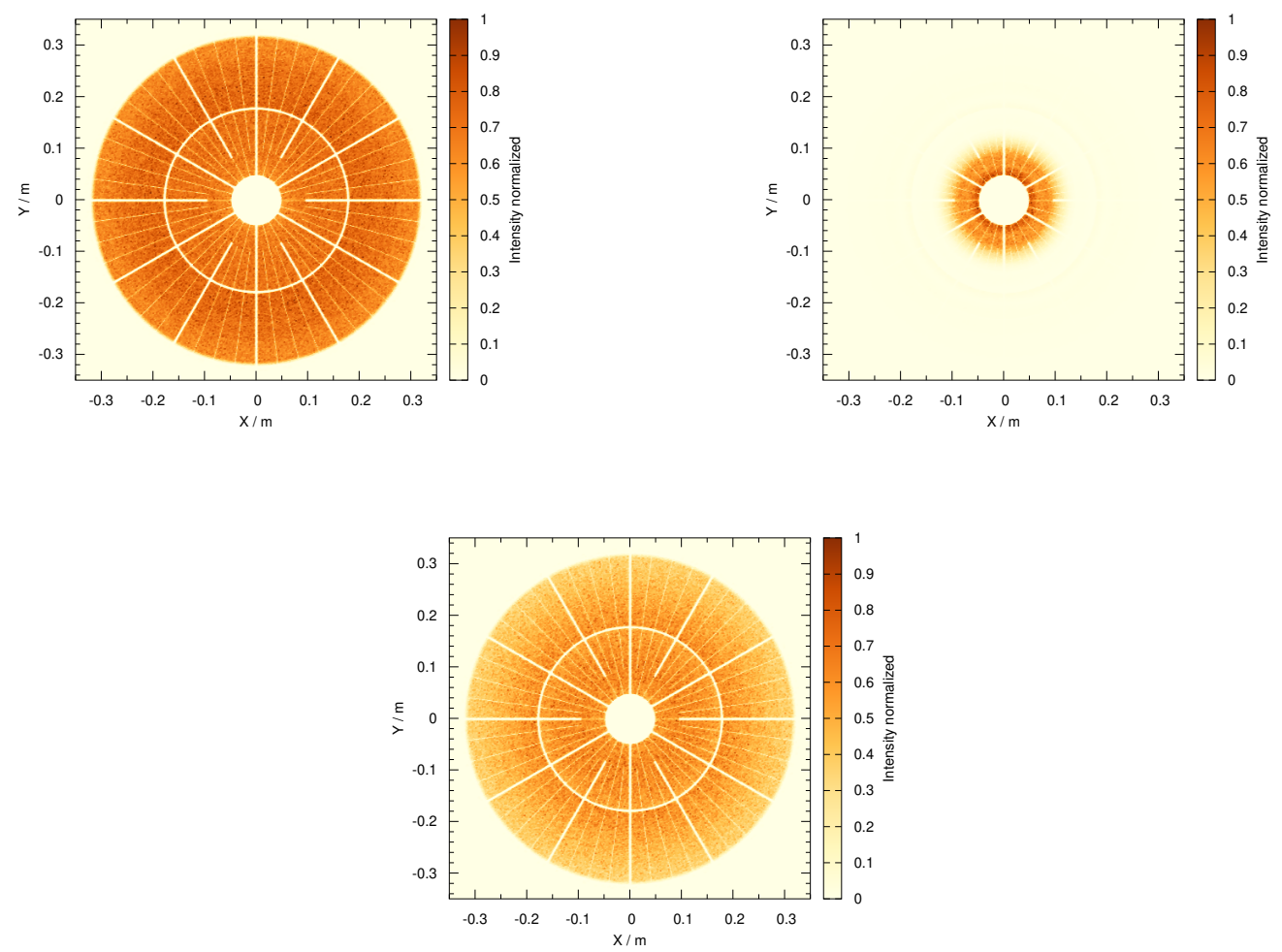

Figure 10. Intensity distribution directly after the telescope optics for the BabyIAXO (as proposed) model at two discrete energies: $2 \mathrm{keV}$ (top left), $12 \mathrm{keV}$ (top right), and for a representative spectrum (bottom).

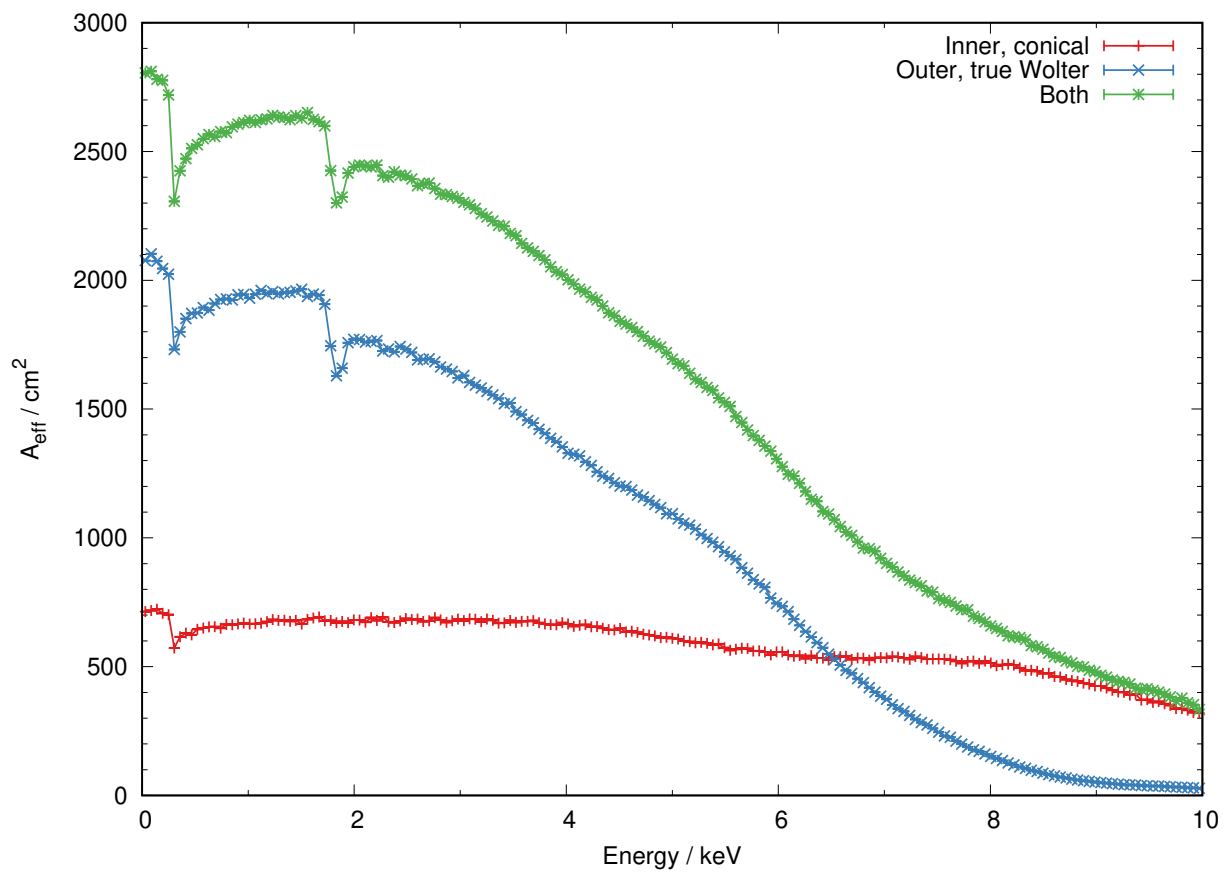

Figure 11. Effective area as a function of energy for our experimental version of the proposed BabyIAXO solar telescope. 
All simulations reported in this paper have been run on a standard desktop computer, and within the timeframe of $<3 \mathrm{~min}$. For the procedure outlined in section 2.4.1, a necessary requirement is that the phase space in sufficiently sampled. This becomes a limiting factor for large phase spaces. We further speculate that an important aspect of telescope simulation should be background estimation. In a ray tracing context, this requires allowing branching of ray paths, which in turn leads to a larger simulation phase space to be sampled. In this sense computational efficiency is a real concern, towards which considerable effort is invested, e.g. as reported in ref. 12 .

In summary, we have in this article reported some snapshots of simulation models performed with the AstroX add-on toolbox for McXtrace, and shown that it may be used for accurate ray-tracing simulations of telescope optics, with a reasonably small effort.

\section{REFERENCES}

1. H. Wolter, "Spiegelsysteme streifenden Einfalls als abbildende Optiken für Röntgenstrahlen," Annalen der Physik 10(1-2), 94-114 (1952).

2. R. C. Chase and L. P. VanSpeybroeck, "Wolter-Schwarzschild Telescopes for X-Ray Astronomy," Applied Optics 12(5), 1042 (2008).

3. S. Chen, S. Ma, and Z. Wang, "Wolter-I-like X ray telescope structure using one conical mirror and one quadric mirror," Chin. Opt. Lett. 14, 123401 (2016).

4. J. E. Harvey, A. Krywonos, P. L. Thompson, et al., "Grazing-incidence hyperboloid-hyperboloid designs for wide-field x-ray imaging applications," Applied Optics 40(1), 136-144 (2001).

5. E. Bergbäck Knudsen, A. Prodi, J. Baltser, et al., "McXtrace : a Monte Carlo software package for simulating X-ray optics, beamlines and experiments," Journal of Applied Crystallography 46, 679-696 (2013).

6. A. Abeln, K. Altenmüller, S. A. Cuendis, et al., "Conceptual design of BabyIAXO, the intermediate stage towards the international axion observatory," (2020).

7. The Nuclear Spectroscopic Telescope Array science operations center, "NuSTAR," (2020).

8. Chandra X-ray Center, "Chandra X-ray Observatory - NASA's flagship X-ray telescope," (2020).

9. X. Barcons, D. Barret, A. Decourchelle, et al., "Athena: ESA's X-ray observatory for the late 2020s," Astronomische Nachrichten 338, 153-158 (2017).

10. M. J. Collon, G. Vacanti, R. Günther, et al., "Silicon pore optics for the ATHENA telescope," in Space Telescopes and Instrumentation 2016: Ultraviolet to Gamma Ray, 9905, 990528, SPIE (2016).

11. J. C. E, A. Hafner, T. Kluyver, et al., "VINYL: The VIrtual Neutron and x-raY Laboratory and its applications," in Advances in Computational Methods for X-Ray Optics V, O. Chubar and K. Sawhney, Eds., 11493, 190 - 200, International Society for Optics and Photonics, SPIE (2020).

12. E. Knudsen, P. Willendrup, J. Garde, et al., "McXtrace anno 2020 - Complex sample geometries and GPU acceleration," in Proceedings of SPIE - The International Society for Optical Engineering, 11493 (2020).

13. D. Spiga, F. Christensen, M. Bavdaz, et al., "Simulation and modeling of silicon pore optics for the ATHENA X-ray telescope," in Proceedings of SPIE - The International Society for Optical Engineering, 9905 (2016).

14. J. R. P. Angel, "Lobster Eyes As X-Ray Telescopes," in Space Optics Imaging X-Ray Optics Workshop, 0184, 84-85, SPIE (1979).

15. A. G. Peele, K. A. Nugent, A. V. Rode, et al., "X-ray focusing with lobster-eye optics: a comparison of theory with experiment," tech. rep. (1996).

16. F. A. Harrison, W. W. Craig, F. E. Christensen, et al., "The Nuclear Spectroscopic Telescope Array (NuSTAR) high-energy X-ray mission," Astrophysical Journal 770(2) (2013).

17. C. J. Hailey, H. An, K. L. Blaedel, et al., "The Nuclear Spectroscopic Telescope Array (NuSTAR): optics overview and current status," in Space Telescopes and Instrumentation 2010: Ultraviolet to Gamma Ray, 7732, 77320T, SPIE (2010).

18. K. K. Madsen, F. A. Harrison, C. B. Markwardt, et al., "Calibration of the NuSTAR high-energy focusing X-ray telescope," Astrophysical Journal, Supplement Series 220 (2015).

19. K. Madsen, B. W. Grefenstette, H. Miyasaka, et al., "Effective area calibration of the nuclear spectroscopic telescope array (NuSTAR)," 67, SPIE-Intl Soc Optical Eng (2018). 
20. N. J. Westergaard, "MT_RAYOR: a versatile raytracing tool for x-ray telescopes," in Optics for EUV, $X$-Ray, and Gamma-Ray Astronomy V, 8147, 81470Y, SPIE (2011).

21. K. K. Madsen, F. A. Harrison, P. H. Mao, et al., "Optimizations of Pt/SiC and W/Si multilayers for the Nuclear Spectroscopic Telescope Array," in Optics for EUV, X-Ray, and Gamma-Ray Astronomy IV, $\mathbf{7 4 3 7}$, 743716, SPIE (2009).

22. Jupyter Team, "Jupyter project documentation," (2015).

23. D. L. Windt, "IMD-Software for modeling the optical properties of multilayer films," tech. rep. (1998).

24. "The Chandra Proposers' Observatory Guide," (2019).

25. R. Willingale, G. W. Fraser, and J. F. Pearson, "Optimization of square pore optics for the x-ray spectrometer on Bepi-Columbo," in Optics for EUV, X-Ray, and Gamma-Ray Astronomy II, 5900, 590012, SPIE (2005).

26. M. J. Bradshaw, V. Burwitz, G. Hartner, et al., "Developments in testing x-ray optics at MPE's PANTER facility," 42, SPIE-Intl Soc Optical Eng (2019).

27. J. I. Robledo, C. A. Pérez, and H. J. Sánchez, "A compact high-resolution spectrometer based on a segmented conical crystal analyzer," Review of Scientific Instruments 91(4), 043105 (2020).

28. D. Prokopiou, J. McGovern, G. Davies, et al., "A new parafocusing paradigm for X-ray diffraction," Journal of Applied Crystallography 53(4) (2020). 